\title{
Sobre o acolhimento: discurso e prática em Unidades Básicas de Saúde do município do Rio de Janeiro
}

\author{
About the reception: discourse and practice in the Basic Health Units \\ in the city of Rio de Janeiro \\ Tarciso Feijó da Silva1, Valéria Ferreira Romano ${ }^{2}$
}

RESUMO Este artigo propõe uma análise do acolhimento em Unidades Básicas de Saúde, no município do Rio de Janeiro, que com a utilização de técnicas de observação sistemática e entrevista semiestruturada construiu sentidos sobre esse acolhimento. No dito, os profissionais o consideram como tecnologia para ampliação da escuta e diminuição da fragmentação do cuidado. No entanto, na prática constroem peregrinação de usuários em busca do cuidado e frágil trabalho em equipe no desenho do acolhimento proposto. Percebeu-se dificuldade de incorporar na prática os conceitos de longitudinalidade e coordenação do cuidado, associada à postura e ao envolvimento dos profissionais com o acolhimento.

PALAVRAS-CHAVE Acolhimento; Atenção Primária à Saúde; Estratégia Saúde da Família.

ABSTRACT This article intends to do an analysis about the reception in Basic Health Units in the city of Rio de Janeiro, which by the means of techniques of systematic observation and semi-structured interview, built senses about the reception. Then, professionals consider reception as a technology for the increasing of the listening and the decreasing of the fragmentation of the cared one. However, in practice they build pilgrimage for the users in the pursuit of care and fragile teamwork to go along with the proposed reception. It' was noticed difficulty in incorporating in practice the concepts practice of longitudinality and coordination of the care, associated to the posture and involvement of professionals with the reception.

KEYWORDS User embracement; Primary Health Care; Family Health Strategy.

1 Prefeitura do Rio de Janeiro, Estratégia Saúde da Família - Rio de Janeiro, Brasil. Universidade Estadual do Rio de Janeiro (Uerj), Policlínica Piquet Carneiro - Rio de Janeiro, Brasil.

tarcisofeijo@yahoo.com.br 


\section{Introdução}

O acolhimento evoca um sentido de urgente atualidade na Atenção Primária à Saúde (APS), na qual assume uma postura polissêmica, uma vez que agrega múltiplos discursos e práticas na saúde. Abordando alguns desses sentidos, observa-se que ele pode ser entendido tanto como elemento inicial do processo de trabalho em saúde, assumindo conexões que acionam as tecnologias leves (MERHY ET AL., 1997), como também pode assumir um lugar de aplicação de diretrizes operacionais para a materialização dos princípios do Sistema Único de Saúde (SUS), em particular a integralidade, a universalidade e a equidade (PINHEIRO, 2001).

Quase que intuitivamente, o acolhimento proporciona em nós uma representação que reporta para compreendê-lo como um momento de encontro mediado pela escuta e pelo vínculo, o que aciona um sentido de disponibilidade de tempo e compromisso para a sua realização. No entanto, considerando o processo de trabalho na atenção primária, Silva et al. (2005) percebem o uso do acolhimento como uma estratégia para atendimento à demanda espontânea; enquanto Guisardi e Fracolli (2005) o abordam como um instrumento para mudanças nesse mesmo processo. Não estamos aqui defendendo a ideia de que trabalhar com demanda espontânea implica em não se disponibilizar a oferecer tempo e compromisso na escuta, mas, pelo contrário, provocamos o leitor a pensar sobre $o$ acolhimento considerando-o como possibilidades, seja no atendimento agendado, seja na demanda espontânea de Unidades Básicas de Saúde (UBS). Assim, alguns autores, como Poli e Norman (2006), o assumem como um arranjo institucional de difícil execução, já que se propõe a trabalhar a demanda espontânea, a ampliação do acesso e a concretização da missão institucional da APS como principal porta de entrada do SUS.
A Política Nacional de Atenção Básica (PNAB) (BRASIL, 2011) recomenda não só a estruturação de um serviço de saúde organizado para assumir sua função central de acolher, escutar e oferecer uma resposta positiva capaz de resolver a grande maioria dos problemas de saúde da população, e/ou de minorar danos e sofrimentos, como também se responsabilizar pela resposta ao usuário, ainda que ofertada para outros pontos de atenção da rede. Assim, relacionamos o acolhimento com gestão do cuidado e processo de trabalho, entendendo-o como múltiplo e singular em sua dimensão de produção da saúde.

Dessa forma, o entendemos não apenas como uma forma humanizada, comprometida e respeitosa e lidar com a relação profissional-usuário (BRASIL, 2008), mas também como um recurso de garantia do acesso aos serviços disponíveis para atendimento às necessidades de saúde da população (FRANCO ET AL., 1999). Nessa perspectiva, acesso e acolhimento articulam-se valorizando o cuidado como central no processo de trabalho na APS; mas de que cuidado estamos falando? Segundo Boff (1999):

O que se opõe ao descuido e ao descaso é o cuidado. Cuidar é mais que um ato; é uma atitude. Portanto, abrange mais que um momento de atenção. Representa uma atitude de ocupação, preocupação, de responsabilização e de envolvimento afetivo com o outro. (BOFF, 1999, P. 3)

O modelo de formação em saúde aciona menos o sentido de cuidado e mais o sentido de cura, focando em uma abordagem biológica como resposta absoluta e secundarizando a subjetividade como estrutural. A necessidade de orientação do processo de trabalho pelas relações que são estabelecidas durante o encontro entre profissionais e usuários poderia ser potencializada se mediada por uma escuta, que permite acionar as necessidades do usuário e construir vínculos (BRASIL, 2005). 
O acolhimento, nesse contexto, deveria ser comum a todos os profissionais na atenção primária. Franco (1999), nos convida a olhar o momento acolhimento:

Olhando esses momentos - pelo lado do trabalho tanto do médico, quanto do de um porteiro de um serviço de saúde - são-nos reveladas questões-chave sobre os processos de produção em saúde, nos quais o acolhimento adquire uma expressão significativa. Isto é, em todo lugar em que ocorre um encontro enquanto trabalho de saúde - entre um trabalhador e um usuário, operam-se processos tecnológicos (trabalho vivo em ato) que visam à produção de relações de escutas e responsabilizações, as quais se articulam com a constituição dos vínculos e dos compromissos em projetos de intervenção. Estes, por sua vez, objetivam atuar sobre necessidades em busca da produção de algo que possa representar a conquista de controle do sofrimento (enquanto doença) e/ou a produção da saúde. (FRANCO, 1999, P. 1).

Se a capacidade resolutiva está diretamente relacionada ao acolhimento realizado e ao compromisso dos profissionais, ele pode ser pensado como um instrumento de trabalho que oportuniza a produção do cuidado (FRACOLLI ET AL., 2003). Pensado dessa forma, entender a dinâmica de funcionamento de uma unidade de saúde na atenção primária, contribuiria para compreender a regulação dos fluxos e das linhas de cuidado, ajudaria a realizar um diagnóstico e levantamento das principais demandas de atendimento e, ainda, permitiria um direcionamento do usuário pela rede de atenção em saúde, visando à integralidade do cuidado.

Recentemente, têm sido discutidos diversos modos de se organizar os serviços de saúde, assim como de que forma o cuidado em saúde pode ser operacionalizado. Normativas de práticas de produção do cuidado em saúde têm sido projetadas com o objetivo de atender a diferentes demandas (BRASIL, 2004).
O que percebemos é a prevalência da singularidade do cuidado, na medida em que na prática dos serviços os problemas de saúde, por mais que sejam semelhantes, tendem a ter diferentes repercussões, considerando os modos de viver, de agir e as condições socioeconômicas de cada usuário. A APS, como porta de entrada preferencial, precisa, para responder a cada uma dessas demandas, desenvolver mecanismos de escuta, pautada em critérios de vulnerabilidade e necessidade.

Em 2004, o Ministério da Saúde lançou a Política Nacional de Humanização (PNH), a qual estabeleceu parâmetros para cada ponto da Rede de Atenção à Saúde (RAS), visando atendimento das necessidades dos usuários. Como parâmetro para a atenção primária, a PNH (BRASIL, 2004) definiu:

Elaboração de projetos de saúde individuais e coletivos para usuários e sua rede social, considerando as políticas intersetoriais e as necessidades de saúde; incentivo às práticas promocionais da saúde; formas de acolhimento e inclusão do usuário que promovam a otimização dos serviços, o fim das filas, a hierarquização de riscos e 0 acesso aos demais níveis do sistema. (BRASIL, 2004, P. 13).

A Organização Mundial de Saúde (OMS, 2008), concluiu que o acolhimento tem a capacidade de medir as expectativas dos cidadãos em relação à forma como gostariam de ser tratados pelos sistemas de serviços de saúde. De acordo com a publicação, podemos avaliar o acolhimento considerando: respeito pela dignidade das pessoas; confidencialidade, ou seja, direito de decidir quem pode ter acesso às informações sobre o seu estado de saúde; autonomia para participar de escolhas relativas à sua própria saúde; direito a uma atenção pronta; direito a serviços de saúde adequados; liberdade de escolha do prestador dos serviços de saúde; direito a receber serviços de saúde providos de forma segura, de modo a evitar procedimentos médicos impróprios e acesso a redes 
de proteção social, como amigos e famílias, pelas pessoas que estão sendo acolhidas pelo sistema de serviços de saúde.

Considerando o exposto - e fruto de uma dissertação de Mestrado em Atenção Primária com Ênfase na Estratégia Saúde da Família, realizado na Fiocruz - este artigo pretende analisar dinâmicas de acolhimento observadas em duas UBS, no município do Rio de Janeiro.

\section{Metodologia}

Este artigo apresenta o resultado de uma pesquisa de campo, de base qualitativa, que realizou um estudo descritivo e exploratório em duas UBS no município do Rio de Janeiro: uma Clínica da Família (CF) e um Centro Municipal de Saúde (CMS), entre os meses de julho e agosto de 2013; sendo aprovado pelo Comitê de Ética em Pesquisa da Ensp/ Fiocruz sob o ${ }^{\circ} 182.519$ e recebido anuência institucional da Secretaria Municipal de Saúde do Rio de Janeiro.

Nesse município, segundo a Carteira de Serviços da Atenção Primária à Saúde (SMSDC, 2011), as UBS são divididas de acordo com o perfil de atendimento, sendo assim classificadas: Módulo A: Clínica da Família, seguindo o processo de trabalho da Estratégia Saúde da Família; Módulo B: Centro Municipal de Saúde, seguindo o processo de trabalho dos tradicionais Postos de Saúde, com presença de especialistas.

A pergunta que motivou o estudo foi: como vem sendo realizado o acolhimento nas UBS no município do Rio de Janeiro, considerando a Politica Nacional de Humanização, a Política Nacional de Atenção Básica e a Carteira de Serviços do município?

Os sujeitos da pesquisa foram, portanto, os próprios trabalhadores da saúde, em um universo de 22 profissionais pesquisados, a saber: médico, enfermeiro, dentista, técnico de enfermagem, técnico de higiene dental, Agente de Vigilância em Saúde (AVS),
Agente Comunitário de Saúde (ACS), auxiliar de portaria, auxiliar de serviços gerais, um profissional do Núcleo de Apoio a Saúde da Família (Nasf) e o gerente de ambas as unidades. Foram realizadas 11 entrevistas por unidade de acordo com as categorias apresentadas.

Os critérios de inclusão para escolha dos profissionais foram aplicados nas duas UBS pesquisadas, a saber: tempo de atuação na atenção primária, especificamente na Estratégia Saúde da Família (ESF); possuir especialização na área da atenção primária, quando profissional médico, enfermeiro e dentista, e outros cursos específicos relacionados à atenção primária ou saúde da família, quando profissionais de nível médio.

A pesquisa foi realizada de acordo com as seguintes fases: estudo bibliográfico (MINAYO, 2007), observação sistemática (BECKER, 1997) e entrevista semiestruturada (MINAYO, 2007). O estudo bibliográfico consistiu de uma pesquisa na base de dados utilizando os descritores: acolhimento, atenção primária e ESF, nos atualizando sobre como o acolhimento tem sido utilizado na ESF de maneira sistemática e nem sempre adequada a sua proposta original de produzir escuta qualificada.

A observação sistemática foi realizada como apoio às entrevistas e permitiu identificar a configuração do acolhimento nas unidades, apontando fragilidades no processo de trabalho, o que será melhor explorado adiante. Foram realizados dois turnos de observação sistemática em cada unidade, com um roteiro predefinido, perfazendo um total de 20 horas de observação.

A entrevista semiestruturada foi realizada para identificar os diversos significados das falas dos profissionais das diferentes categorias no que tange ao acolhimento e suas implicações para a atenção primária. Além disso, possibilitou identificar a compreensão por parte dos profissionais sobre a importância do acolhimento para a organização do processo de trabalho nas unidades. Um roteiro previamente elaborado, com os 
mesmos dados utilizados posteriormente na pesquisa, conduziu as entrevistas, e uma entrevista piloto foi realizada, o que permitiu avaliar a viabilidade dos instrumentos de pesquisa.

À análise do diário de campo, registro da observação sistemática, foi realizado contrapondo as informações coletadas com as informações dos documentos oficiais, e esse exercício de reflexão nos trouxe amadurecimento sobre o tema. Assim, para a análise das entrevistas, utilizamos Bardin (1977) como referência quando realizamos uma leitura flutuante, e identificamos termos específicos que respondiam à questão e ao objetivo do estudo. Esses termos foram agrupados em três categorias analíticas: o acolhimento na visão dos profissionais, implicação dos profissionais com o acolhimento proposto e importância do acolhimento para o processo de trabalho das unidades. A análise dessas categorias permitiu identificar o envolvimento dos profissionais com o acolhimento proposto, conceitos relacionados ao acolhimento e sua relevância para o processo de trabalho.

A maioria dos entrevistados possuía mais de um ano de atuação em ambas as unidades, não possuíam formação específica em saúde da família, embora tivessem especialização em áreas relacionadas à saúde pública, e cumpriam carga horária semanal de 40 horas, à exceção de alguns entrevistados, que possuíam especialização ou residência em saúde da família (gerentes das duas unidades, um profissional médico da $\mathrm{CF}$, um enfermeiro do CMS e um profissional do Nasf do CMS).

No que concerne ao regime de contratação, na CF apenas o AVS era estatutário, e todos os outros trabalhavam em regime de contrato CLT (Consolidação das Leis do Trabalho); já no CMS, havia profissionais com distintos vínculos de trabalho.

O curso introdutório - requisito para atuação na ESF - foi realizado por todos os profissionais que atuavam na ESF; enquanto no CMS, somente os profissionais celetistas que atuavam na ESF haviam realizado o introdutório.

\section{Resultados e discussão}

\section{A exposição da trajetória: potências e fragilidades}

O contato inicial com o campo e com os profissionais, em um primeiro momento, foi realizado sem a identificação prévia do pesquisador, que permaneceu no local pesquisado como observador atento, permitindo realizar escuta, vislumbrar posturas e o manejo dos profissionais durante o atendimento. Assim, o pesquisador pôde identificar um padrão de solidariedade entre os profissionais, gerando potência no processo de trabalho, permeado por momentos de fragilidade. Ou seja, o pesquisador percebeu, nas duas unidades pesquisadas e sem grandes diferenças entre si, uma atitude geral de apoio e tentativa de resolubilidade dos problemas trazidos pelos usuários, apesar de também ter observado atitudes contrárias a estas, sinalizando que nem todos os profissionais e nem todos os serviços de saúde promovem um cuidado esperado.

A competência individual de cada profissional dentro das unidades e a implicação do coletivo com o processo de trabalho não foi uniforme, uma vez que em muitos momentos a desinformação dos profissionais, a falta de espaços de fala e de escuta entre trabalhadores e usuários, contribuíram para a perpetuação de um cuidado descuidado.

Observou-se que os usuários desconheciam o trabalho em equipe desenvolvido na ESF, e poucos valorizavam as práticas de cuidado, priorizando a busca pelo 'exame e o medicamento' nas unidades de saúde. Por outro lado, os trabalhadores tinham dificuldades na reorganização do seu processo de trabalho e muitas vezes não reconheciam as unidades como espaço de exercício da 
micropolítica das relações, distanciando-se da possibilidade de produzir mudanças (ANDRADE ET AL., 2007).

Quando o pesquisador se identificou para os profissionais, eles se empenharam em ofertar possibilidade de compreensão sobre o envolvimento e entendimento da importância do acolhimento para a organização do processo de trabalho nas unidades, gerando como consequência, um reconhecimento dos serviços e fluxos de acolhimento adotados.

Nas duas unidades, de acordo com o modelo de atenção e a estrutura física, existiam fluxos que direcionavam o acesso aos serviços. Foi possível identificar similaridades relacionadas à postura e ao comportamento dos profissionais e usuários de acordo com a demanda de atendimento.

\section{Descrevendo o acolhimento}

O acolhimento era realizado na entrada das unidades, nos corredores e em salas específicas, onde um profissional avaliava a necessidade imediata ou não de atendimento. Havia escala de revezamento para determinado grupo de profissionais que assumiam a responsabilidade por receber e direcionar os usuários dentro das unidades, sendo possível observar quem trabalhava e como trabalhavam os profissionais nessas unidades, bem como que condições de trabalho tinham.

$\mathrm{O}$ acolhimento nas duas unidades observadas estava organizado para possibilitar a escuta dos motivos que levavam o indivíduo a procurar o serviço de saúde naquele momento. No entanto, os usuários chegavam solicitando que seus problemas de saúde fossem resolvidos sempre de imediato, evidenciando rotas de fuga ao instituído, em que os usuários demonstravam que a dinâmica do serviço não fazia parte dos seus saberes, ou necessidades. Gerar movimentos que tornem os profissionais conhecedores dessa visão e reconstruir, de forma dialógica, a concepção com os usuários fazendo parte do processo de acolhimento nos parece ser uma saída fundamental (MACEDO ET AL., 2011).

\section{Atravessando o olhar}

Certamente, uma das limitações da pesquisa geradora deste artigo, apoia a ideia de que talvez tivéssemos que analisar as duas UBS em separado, considerando que possuem trajetórias e tempos de existência diversos. No entanto, como a coexistência de modelos foi uma estratégia utilizada no município do Rio de Janeiro para a expansão da APS, achamos fundamental observar e registrar tal realidade, mesmo que parecesse aparentemente ambígua.

Assim, foi possível observar um processo relacional denso e ruídos na comunicação entre os profissionais do CMS, uma vez que havia dificuldade de integração entre eles, gerando uma gestão pouco compartilhada. Tais fatores, aparentemente, produziam certa insatisfação entre os profissionais estatutários que não possuíam entendimento claro sobre o processo de trabalho relacionado à atuação dos profissionais celetistas na ESF.

Em contrapartida, os profissionais celetistas pareciam sentir-se sobrecarregados e desmotivados e cobravam maior envolvimento dos profissionais estatutários da unidade no processo de trabalho.

$\mathrm{Na}$ CF, a postura do AVS - estatutário que atuava junto à ESF com atribuição de identificar e desenvolver ações específicas para a diminuição de focos que poderiam contribuir para aparecimento de doenças endêmicas - era de pouco envolvimento com outras questões que não estivessem relacionadas à sua função específica, sugerindo manutenção da fragmentação do processo de trabalho.

Nas duas unidades, os profissionais auxiliares de portaria e auxiliar de serviços gerais contribuíam para a regulação do acesso direcionado os usuários, uma vez que era fácil encontrá-los na entrada e corredores. Muitas vezes, observamos a atuação desses 
profissionais mediando a 'primeira escuta' aos usuários, fazendo supor que, em um serviço de saúde todos os profissionais fazem clínica, não no sentido médico, mas de envolvimento intenso com as questões trazidas pelos usuários.

Todos os ACS possuíam alguma identificação - ou estavam uniformizados, ou com crachá institucional - permaneciam na porta de entrada ou na recepção das unidades e atuavam como facilitadores do acesso, organizando a porta de entrada, evitando acúmulo de usuários e fila de espera para atendimento. Na recepção, recebiam os usuários de acordo com a sua equipe de referência. Nas falas dos profissionais durante as entrevistas, o facilitador do acesso foi identificado como 'posso ajudar'.

Entre as atividades desenvolvidas pelos ACS, foi possível observar: escuta, identificação da demanda, direcionamento para setores específicos, visando a realização de algum procedimento, e encaminhamento para sala da unidade específica, onde o enfermeiro realizava avaliação de risco dos usuários egressos por demanda espontânea.

A exemplo do que é feito nos protocolos de classificação de risco (BRASIL, 2012) utilizados nos serviços de urgência, as situações não agudas e os três tipos de atendimento de situações agudas ou crônicas agudizadas, para fins de visualização e comunicação, eram representadas por cores e orientavam os enfermeiros, possibilitando a classificação de risco na atenção primária. A utilização de um espaço específico para atendimento da demanda espontânea com a classificação de risco descrita contribuía para a organização do processo de trabalho nas unidades, uma vez que havia um fluxo predeterminado para esse tipo de demanda.

Foi possível observar, e nos parece importante destacar, que a maior parte da demanda que as unidades absorviam não apresentava quadro agudizado e buscava o atendimento por necessidade de acompanhamento, agendamentos prévios ou referenciadas de outros pontos da rede.

\section{As falas: acolhimento prescrito e acolhimento real}

$\mathrm{Na}$ análise das entrevistas, buscou-se identificar os diversos sentidos atribuídos ao acolhimento pelos profissionais, a implicação destes com o processo de acolher e a sua compreensão sobre a importância do acolhimento para o processo de trabalho das unidades. Assim, ele foi apontado como dispositivo para atendimento da demanda espontânea e garantia de acesso dos usuários aos serviços. O acolhimento foi relatado como organizador da porta de entrada na atenção primária, permitindo melhor direcionamento para os serviços que a unidade disponibiliza, assim como para outros pontos de atenção da rede; além de parecer possuir grande capacidade resolutiva quando atrelado a uma escuta qualificada.

Vejamos as falas abaixo:

Uma das coisas mais importantes do acolhimento é poder direcionar aquele paciente atendendo a sua demanda. Eu acho que pode contribuir muito para organização dessa maneira informando bem, evitando que o paciente tenha que ir aos diversos setores com a mesma pergunta tendo respostas diferentes. (NASF).

Bem, o acolhimento é a entrada do paciente na nossa unidade. É a partir dali que a gente vai direcionar essa clientela para o atendimento de acordo com a necessidade dele. (ENF).

A partir do acolhimento nós podemos realizar uma escuta qualificada procurando ver a real necessidade do usuário que chega até a unidade de saúde. A partir dessa escuta qualificada a gente também vai estar orientando o usuário para qual profissional ele vai ser assistido. (GERENTE).

APNAB (BRASIL, 2012) estabelece que o serviço de saúde deve se organizar para assumir sua função central de acolher, escutar e oferecer uma resposta positiva, capaz de resolver a grande maioria dos problemas de saúde da 
população e/ou de minorar danos e sofrimentos desta, ou ainda se responsabilizar pela resposta, ainda que esta seja ofertada em outros pontos de atenção da rede. O profissional médico reconhece a importância do exposto na seguinte fala:

[...] ao ajudar na organização de todo o sistema de saúde dentro dessa nova lógica de promoção da saúde, prevenção de doenças e de educação em saúde, não esquecendo a parte curativa. (MED).

Entender o acolhimento dentro dessa lógica de promoção e prevenção da saúde, não esquecendo a parte curativa, exige envolvimento dos profissionais e entendimento da necessidade de longitudinalidade do cuidado. Para isso, ele deve se iniciar desde a recepção, que é a porta de entrada da unidade, mas não deve ser reduzido a ela, pois como diretriz clínica se destina à organização do serviço e do processo de trabalho, na garantia do acesso qualificado, resolutivo, baseado na construção do vínculo, tendo como princípio a integralidade do cuidado (SMSDC, 2011). "O acolhimento é uma grande ferramenta aonde a gente pode através dele direcionar, organizar e planejar. Porque ele também nos traz um diagnóstico" (GERENTE).

Um acolhimento bem feito direciona o cadastrado dentro da unidade. Ele entende como é que a unidade funciona e não fica perdido. Entende o fluxo da unidade. Porque às vezes a pessoa vem querendo procurar informação, não sabe como é que funciona a clínica da família, como é que é a clínica da família. Não sabe onde é o curativo, a vacina. Se ele tiver esse direcionamento, esse acolhimento ele consegue se mobilizar. (NASF).

Se acolher implica singularizar - entender a demanda ou necessidade do usuário como algo individual - envolver-se com o processo, com o trabalho proposto, parece ser uma evidente necessidade; principalmente quando associamos a capacidade resolutiva do acolhimento à capacidade de escuta do profissional e à sua capacidade de resolver ou de buscar respostas para as demandas encontradas.

Assim, ficou claro que o acolhimento dito foi, em algumas situações, diverso do praticado, já que nem sempre foi executado como um modo de operar os processos de trabalho em saúde, de forma a atender a todos que procuram os serviços de saúde, ouvindo seus pedidos e assumindo no serviço uma postura capaz de dar respostas. No entanto, as falas dos profissionais parecem ser assertivas em relação aos sentidos amplos e simultaneamente ambíguos que atribuem:

O acolhimento é todo um processo que pode ser resolvido facilmente mais que por ser um fator que conta com o pessoal de cada um, com o caráter e a boa vontade, a personalidade de estar ali disposto a ajudar acaba virando um nó. (TÉC. ENF).

Se tiver um acolhimento organizado para todos os serviços da clínica. Se este processo estiver todo organizado o paciente não perde tempo ele não roda a clínica inteira para poder procurar quem é a pessoa certa, qual o local que ele tem que ir. (ADM).

A PNH (BRASIL, 2004) enfatiza que o acolhimento não é um espaço ou um local, mas uma postura ética: não pressupõe hora ou profissional específico para fazê-lo, implica compartilhamento de saberes, angústias e invenções, tomando para si a responsabilidade de 'abrigar e agasalhar' outrem em suas demandas, com responsabilidade e resolutividade sinalizada para cada caso.

Já a PNAB (BRASIL, 2012) atribui a todos os membros da equipe a participação no acolhimento dos usuários, realizando a escuta qualificada das necessidades de saúde, procedendo à primeira avaliação (classificação de risco, avaliação de vulnerabilidade, coleta de informações e sinais clínicos) e identificação das necessidades de intervenções de cuidado, proporcionando atendimento humanizado, responsabilizando-se pela 
continuidade da atenção e viabilizando o estabelecimento do vínculo.

$\mathrm{Na}$ prática, no entanto, percebeu-se através das entrevistas e observação realizadas uma sobrecarga de trabalho do profissional ACS que assume o papel de acolher os usuários na recepção da unidade, nas visitas domiciliares, nos grupos e ações realizados e que ainda atua como facilitador do acesso, organizando a demanda de atendimento dos que procuram o serviço diariamente.

Os profissionais reiteravam nas unidades um acolhimento dependente de um espaço físico, o que fragilizava o cuidado, na medida, em que não se permitia criar construção de uma relação de confiança e compromisso dos usuários com as equipes e os serviços (MACEDO ET AL., 2011).

"Tem sempre um agente de saúde e um posso ajudar atendendo. Eles é que entendem melhor este processo" (THD).

Nessa unidade aqui quem faz o acolhimento são os ACS. Eles que dão tanto informação para saúde da família como para o posto. Então a gente é obrigada a saber tudo. Saber como funciona o posto e saber como funciona a clínica da família. (ACS).

O acolhimento é complicado porque ninguém gosta de ficar no acolhimento bem na verdade. $O$ posso ajudar então pior ainda. Mais dependendo do agente que ta ali esse paciente pode ser ouvido porque o acolhimento é isso é escuta. (NASF).

Eles passam pelas baias na recepção para o agente de saúde de referência da equipe. A princípio nós temos também um agente de saúde escalado diariamente no posso ajudar que a princípio faz a escuta ativa, identifica qual a necessidade desse indivíduo. (GERENTE).

As falas referidas pelos profissionais corroboram a identificação de um acolhimento centrado no ACS e com pouco envolvimento por parte dos outros profissionais.
A necessidade de contato com o profissional ACS para legitimização do acesso pode ser evidenciada através das entrevistas e da observação sistemática realizada. O papel que esse profissional desempenha na atenção primária, especificamente, no acolhimento, precisa ser repensado, uma vez que a investigação permitiu reconhecer neles algumas fragilidades, como pode-se observar através da transcrição da fala da ACS:

O acolhimento é escuta. Os ACS são os que mais acolhem os usuários na unidade. Também ficam lá na frente. Daí os usuários logo chegam e perguntam. Só acho que somos os menos preparados para isso. Tivemos um introdutório mais não foi suficiente. Olha quando entrei pra trabalhar aqui pensei que fosse ficar na rua, na casa das pessoas. Mais ficamos muito tempo aqui dentro. Daí as pessoas reclamam e com razão. No acoIhimento tudo é muito difícil. Por exemplo, minha equipe está sem médico no momento. O paciente quer médico, quando chega e digo que não tem ele logo fica grosseiro e pergunta o que eu estou fazendo ali. $O$ acolhimento funciona, a gente ta ali, atendendo o paciente quer orientação à gente dá, quando quer vacina, curativo a gente diz qual é a sala. Mas e quando ele quer médico? $O$ que a gente faz? Se tivesse médico em todas as equipes, se todos fizessem tudo igualzinho, do mesmo jeito, se tivesse uma ordem acho que o acolhimento seria melhor. Às vezes eu falo uma coisa, quando chega dentro da sala vem o enfermeiro e o técnico de enfermagem e fala outra. Aí o paciente não dá crédito ao que falamos. (ACS).

As fragilidades apontadas pela ACS - "só acho que somos os menos preparados para isso", "tivemos um introdutório mais não foi suficiente", "ficamos muito tempo aqui dentro", "às vezes eu falo uma coisa, quando chega dentro da sala vem o enfermeiro e o técnico de enfermagem e fala outra" e "acho que falta autonomia para gente” - precisam ser sinalizadas, visto que expressam o reconhecimento por parte da profissional da necessidade de valorização e respeito 
pelas atividades que desenvolvem. O que foi apontado pela ACS precisa ainda ser analisado, visando identificar que recursos esse profissional possui para atendimento das demandas pelas quais se torna responsável, considerando sua atuação e as responsabilidades que tem exercido na APS no município do Rio de Janeiro.

\section{Conclusão}

Este artigo aponta para algumas questões importantes que nos remetem a repensar o acolhimento e seu compromisso com o envolvimento e a coordenação do cuidado. Assim, ao propor uma análise sobre o acolhimento no contexto de duas UBS, no município do Rio de Janeiro, que estudadas com a utilização de técnicas de observação sistemática e entrevista semiestruturada, construiu sentidos sobre o acolhimento, advindos dos profissionais das unidades pesquisadas. Mais do que tudo, esta análise propôs também certa incorporação do que denominamos de 'possibilidades', ou seja, sem ser prescritivo ou propositivo, intencionamos, mais provocar e deixar perguntas do que ofertar respostas.

Retomando o argumento das possibilidades, uma delas foi a de compreender que, no dito, os profissionais consideraram o acolhimento como tecnologia para ampliação da escuta e diminuição da fragmentação do cuidado. No entanto, no realizado, na prática, no cotidiano do trabalho, ainda ofertaram peregrinação de usuários em busca do cuidado, excesso de atribuições para os ACS e frágil trabalho em equipe para respaldar o acolhimento proposto.

Revelaram-se, portanto, fragilidades relacionadas à conformação, postura, envolvimento e comprometimento dos profissionais, que ao demonstrarem certa dificuldade em incorporar os conceitos de longitudinalidade e coordenação do cuidado, geraram, paradoxalmente, aumento da demanda e pouca resolubilidade.
Sendo assim, possibilidades outras, com potencial motivador, será que poderíamos supor uma reconstrução teórico-metodológica sobre a produção do cuidado, utilizando o acolhimento como referência?

Ainda como possibilidade assinalada, trouxemos o modo como cada profissional estava envolvido no processo de acolher e como vinha conduzindo a produção do cuidado, a partir do primeiro contato estabelecido com o usuário, o que nos permitiu inferir que o acolhimento poderia ser um dos principais meios para avaliação da satisfação dos usuários. Principalmente porque ao ser visto como uma importante ferramenta gerencial capaz de regular fluxos e contribuir para organização do processo de trabalho, possui um potencial transformador de práticas.

Considerando a dimensão relacional ocupada pelo acolhimento, vimos que o encontro entre o profissional e o usuário fundamenta a postura dos profissionais, trazendo como exemplo o profissional de nível superior, que pouco se envolveu com o acolhimento em si, fazendo-nos supor que quanto maior a grau de formação dos profissionais maior o seu distanciamento com o acolhimento.

Os ACS e os outros profissionais de nível médio entenderam sua responsabilidade em acolher e direcionar os usuários a partir do primeiro contato, o que ficou evidenciado através de suas falas e da observação realizada. Esses profissionais, quando não tinham capacidade técnica para responder as necessidades dos usuários, demonstravam interesse e empatia em buscar uma resposta.

Por sua vez, os profissionais de nível superior pouco estavam implicados com o acolhimento e assumiam a função de responder de forma pontual às demandas, salvo algumas exceções, quando consideramos o trabalho do enfermeiro na regulação do acolhimento.

A atuação do ACS no acolhimento foi outra possibilidade que mereceu destaque. Esse profissional, nas duas unidades 
estudadas, foi considerado como o principal e primeiro contato do usuário com o serviço. Entretanto, o estudo permitiu observar também, entre outras questões, as limitações na atuação desse profissional e a sua desmotivação relacionada à sobrecarga de trabalho.

Destaque foi dado ao apoio institucional nesse processo. $\mathrm{O}$ envolvimento e a aproximação da gerência com as questões apontadas, reuniões específicas com o corpo técnico das unidades, reuniões periódicas com os ACS, participação nas reuniões de equipe e abertura de espaços de discussão nas reuniões gerais são estratégias que poderiam contribuir para identificação de prioridades e corresponsabilização dos profissionais, já que no dito, o acolhimento foi visto como dispositivo capaz de contribuir para reorganização do processo de trabalho e reconstrução de práticas.

Entre possibilidades, o dito, o realizado e o teorizado, ficamos com as implicações que as falas nos proporcionaram e expectamos contribuir para o debate sobre como motivar, como criar sentidos, como mobilizar os trabalhadores da saúde a apostarem mais no cuidado e menos no descuidado. Lutemos pelo SUS que desejamos!

\section{Referências}

ANDRADE, C. S. et al. Acolhimento: Uma experiência de pesquisa-ação na mudança do processo de trabalho em saúde. Revista APS, Juiz de Fora, v. 10, n. 2, p. 106115, jul./dez. 2007.

BRASIL. Ministério da Saúde. Portaria ${ }^{\circ} 2.488$, de 21 de outubro de 2011. Aprova a Política Nacional de Atenção Básica, estabelecendo a revisão de diretrizes e normas para a organização da Atenção Básica, para a Estratégia Saúde da Família (ESF) e o Programa de Agentes Comunitários de Saúde (PACS). Diário Oficial [da] União. Brasília, DF: Ministério da Saúde, 2011.

Ministério da Saúde. Secretaria de Atenção à Saúde. Departamento de Atenção Básica. Política Nacional de Atenção Básica / Ministério da Saúde. Secretaria de Atenção à Saúde. Brasília, DF: Ministério da Saúde, 2012.

Ministério da Saúde. Secretaria de Atenção à Saúde. Núcleo Técnico da Política Nacional de Humanização. HumanizaSUS: Documento base para gestores e trabalhadores do SUS / Ministério da Saúde, Secretaria de Atenção à Saúde, Núcleo Técnico da Política Nacional de Humanização. 4. ed. Brasília, DF: Ministério da Saúde, 2008.

Ministério da Saúde. Secretaria de Gestão do Trabalho e da Educação em Saúde. Departamento de Gestão da Educação na Saúde. Curso de Formação de Facilitadores de Educação Permanente em Saúde: Unidade de Aprendizagem. Trabalho e relações na produção do cuidado em saúde. Rio de Janeiro: Ministério da Saúde: FIOCRUZ, 2005.

. Ministério da Saúde. Secretaria de Atenção à Saúde. Departamento de Atenção Básica. Política Nacional de Humanização. Brasília, DF: Ministério da Saúde, 2004.

BARDIN, L. Análise de Conteúdo. Lisboa: Editora 70, 1977.

BECKER, H. S. M. Métodos de pesquisa em ciências sociais. São Paulo: Hucitec, 1997.

BOFF, L. Saber Cuidar: Ética do humano, Compaixão pela Terra. Petrópolis: Vozes, 1999.

FRACOLLI, L. A. et al. A visita domiciliar sob o enfoque do acolhimento e sua interface com a abordagem do desmame precoce no programa de saúde da família: um relato de experiência. Revista Eletrônica de Enfermagem, Goiânia, v. 5 n. 2 p. 68-72, 2003.

FRANCO, T. B. et al. O acolhimento e os processos de trabalho em saúde: o caso de Betim, Minas Gerais, Brasil. Cad. Saúde Pública, Rio de Janeiro, v.15, n. 2, p. 345-353, abr./jun. 1999. 
GUISARDI, P. J.; FRACOLLI, L. A. Acolhimento e reorganização das práticas em saúde. Saúde e Sociedade, São Paulo, v. 14, supl. 1, p. 23, 2005.

MACEDO, C. A. et al. Possibilidades e limites do acolhimento na percepção de usuários. Revista Enfermagem, Rio de Janeiro, v. 19, n. 3, p. 457-62, jul./set. 2011.

MERHY, E. E. et al. Em busca de ferramentas analisadoras das tecnologias em saúde: a informação e o dia-a-dia de um serviço, interrogando e gerindo trabalho em saúde. In: MERHY E. E.; ONOCKO R.; (Org.). Agir em saúde: um desafio para o público. São Paulo: Editora Hucitec, 1997, p. 113-50.

MINAYO, M. C. Desafio do Conhecimento: pesquisa qualitativa em saúde. 10 ed. São Paulo: Editora Hucitec, 2007.

ORGANIZAÇÃO MUNDIAL DA SAÚDE (OMS). Relatório Mundial de Saúde. Cuidados Primários em Saúde: agora mais que nunca. Lisboa, 2008.

PINHEIRO, R. As práticas do cotidiano na relação oferta e demanda dos serviços de saúde: um campo de estudo e construção da integralidade. In: PINHEIRO, R.; MATOS, R. A. (Org.).
Os sentidos da integralidade na atenção e no cuidado à saúde. Rio de Janeiro: UERJ, 2001, p. 65-112.

POLI, P; NORMAN, A. H. Acolhimento e (des)medicalização: reflexões sobre essa prática em um Centro de Saúde. In: CONGRESSO BRASILEIRO DE MEDICINA DE FAMÍLIA E COMUNIDADE, 8., Encontro Lusobrasileiro de Medicina Geral, Familiar e Comunitária, 2, 2006, São Paulo. Anais... São Paulo: USP, 2006. p. 246.

SECRETARIA MUNICIPAL DE SAÚDE E DEFESA

CIVIL. Superintendência de Atenção Primária (SMSDC). Superintendência de Atenção Primária. Guia de Referência Rápida. Carteira de Serviços: Relação de serviços prestados na Atenção Primária à Saúde. Rio de Janeiro: SMSDC, 2011. 128 p.

SILVA, R. M. et al. O acolhimento no PSF: da proposta teórica para a operacionalização na prática. Saúde e Sociedade, São Paulo, v. 14, supl. 1, p. 245-246, 2005.

\footnotetext{
Recebido para publicação em outubro de 2014

Versão final em março de 2015

Conflito de interesse: inexistente

Suporte financeiro: não houve
} 